\title{
Respiratory virus infections in immunocompromised patients
}

\begin{abstract}
Respiratory infections caused by viruses such as respiratory syncytial and parainfluenza viruses are often dismissed as trivial - 'it's just a virus' - and in the vast majority of cases in the general population, colds and flu-like illnesses caused by these viruses result in only mild symptoms such as a cough, sore throat, rhinorrhoea, nasal congestion and, less frequently, mild pyrexia. However, in certain groups of patients these viruses are the cause of substantial morbidity and mortality. Immunocompromised patients are one such group and are especially susceptible to disease progression, often with fatal consequences. It is estimated that up to $5 \%$ of all bone marrow transplant patients develop a potentially serious respiratory virus infection [1]. However, most data derive from retrospective studies, or case note documentation. Few prospective studies have been undertaken to determine the incidence of infection and its association with disease.
\end{abstract}

The respiratory viruses implicated in causing severe disease in immunocompromised patients include respiratory syncytial virus (RSV), influenza viruses A and $B$, parainfluenza viruses (especially type 3 [2]) and adenovirus. Rhinovirus has not been included in this list because, although one of the most common causes of upper respiratory tract infection, it is not thought to lead to severe complications in these patients. Similarly, coronaviruses do not seem to cause severe disease in immunocompromised individuals [3].

Infection of an immunocompromised patient with one of the above viruses gives rise initially to upper respiratory tract symptoms similar to those experienced by the general population. Retrospective studies suggest that c. $60-80 \%$ of cases will subsequently develop pneumonia, and of those, $10-40 \%$ overall (up to $80 \%$ in the case of RSV) will die either as a direct or an indirect effect of their respiratory viral infection [1-5]. The median interval between the onset of upper tract symptoms and the development of lower respiratory tract infection is thought to be $c$. 5-6 days.

Unlike influenza virus infection, which may lead to a secondary bacterial pneumonia (often caused by Staphylococcus aureus), RSV infection most commonly results in a primary pneumonitis of purely viral aetiology. Adenovirus differs from the other viruses in that it causes disseminated systemic infection involving not only the respiratory tract, but also the liver, urinary tract and gastrointestinal tract.

Patients at risk of developing severe disease include: preterm infants or those with underlying congenital heart disease or bronchopulmonary dysplasia; those with underlying lung disease such as asthma, chronic obstructive airways disease, and, possibly, cystic fibrosis; and immunocompromised patients, in particular those with impaired cell-mediated immunity. Of the immunocompromised patients, those most at risk are lung transplant and bone marrow transplant patients, as well as leukaemia patients on myeloablative chemotherapy. Liver and kidney transplant patients are thought to be at a lower risk of developing severe disease. Among bone marrow transplant patients, allogeneic transplant confers a higher risk than autologous transplant. Generally, therefore, the greater the level of immunosuppression, the higher the risk [6-9].

Respiratory viruses infecting the upper respiratory tract can often be detected in throat swabs, nasal washings or gargles. A good throat swab, obtained by firmly wiping the back of the throat, is most fruitful. Nasopharyngeal aspiration is preferred in infants. Broncho-alveolar lavage is the specimen of choice for the detection of viruses infecting the lower respiratory tract $[10]$. The number of respiratory virus infections in immunocompromised patients may be underestimated because of the lack of specimens submitted for virological investigation. A lower threshold for performing broncho-alveolar lavage in these patients has been advocated, and it is essential for any lavage obtained from an immunocompromised patient to be tested for respiratory viruses, ideally by a rapid immunofluorescence assay in addition to cell culture.

Immunofluorescence staining of the clinical specimen is the gold standard for rapid laboratory diagnosis. This is usually complemented by virus isolation. Less widespread methods include immuno-enzymatic assays (for RSV antigen) and PCR. Serology provides retrospective information and is often unreliable in immunocompromised patients. Therefore, it is of little value in guiding acute patient management. 
There is a paucity of controlled clinical trial data on antiviral therapy of respiratory virus infections in immunocompromised patients. This has led to a wide diversity of management strategies, and diagnostic protocols for such conditions. RSV infection may respond to tribavirin (ribavirin), and early therapy is likely to be more effective $[3,11-13]$. The outcome of established RSV pneumonia in these patients is poor, regardless of therapy. As upper respiratory tract symptoms often portend lower respiratory tract infections, pre-emptive therapy should be considered [3]. The most effective route of administration of tribavirin is still a subject of controversy, and some recommend a combination of both intravenous and nebulised formulations [3]. Nebulised tribavirin is less well tolerated than the intravenous formulation, and is more often discontinued prematurely because of the occurrence of bronchospasm and pleuritic chest pain. Adenovirus and influenza $\mathrm{A}$ and $\mathrm{B}$ are sensitive to tribavirin in vitro, although clinical data are limited to case reports. Amantadine and rimantadine are effective against influenza type $\mathrm{A}$ infection. Neuraminidase inhibitors such as zanimavir (currently in phase III trials) may also prove effective against influenza viruses in these patients. The therapeutic or prophylactic role of immunoglobulin preparations remains to be ascertained [14].

RSV and parainfluenza viruses are transmitted primarily through inoculation of the mucosal surfaces of the eyes and nose with fingers that have become contaminated through direct contact with an index case, or indirectly via contaminated fomites. In contrast, the primary mode of spread of the influenza and adenoviruses is through respiratory droplets. Most respiratory virus infections in immunocompromised patients probably occur through nosocomial spread $[15,16]$. Infection control measures, such as hand washing, wearing gloves, gowns and face masks, patient isolation and cohort nursing, in conjunction with screening symptomatic patients, may help to prevent spread of infection [17]. In addition, immunocompromised patients may be advised to avoid crowded areas, which are conducive to the spread of respiratory viruses. Amantadine (or rimantadine) prophylaxis, and vaccination (before transplantation) in the case of solid organ transplants, may provide protection against the influenza viruses [18].

Despite improvements in the prevention and management of common respiratory infections in immunocompromised pat-ients, a diagnostic gap remains. There is increasing awareness that viruses such as RSV, parainfluenza, influenza and adenovirus can lead to morbidity and mortality in these patients. However, longitudinal prospective studies are required to assess the true prevalence and incidence in different patient groups. The virological and clinical natural history of these viruses in immunocompromised patients also needs to be established. Most importantly, controlled trials of antiviral agents must be undertaken to demonstrate efficacy of currently available agents.

D. MUIR AND D. PILLAY

Virology Department, Birmingham Public Health Laboratory, Birmingham Heartlands Hospital, Birmingham B9 5ST

E-mail: d.pillay@bham.ac.uk

\section{References}

1. Ljungman P. Respiratory virus infections in bone marrow transplant recipients: the European perspective. $\mathrm{Am} J \mathrm{Med}$ 1997; 102 Suppl: 44-47.

2. Lewis V, Champlin R, Englund $\mathrm{J}$ et al. Respiratory disease due to parainfluenza virus in adult bone marrow transplant recipients. Clin Infect Dis 1996: 23: 1033-1037.

3. Whimbey E, Englund JA, Couch RB. Community respiratory virus infections in immunocompromised patients with cancer. Am J Med 1997; 102 Suppl: 10-18.

4. Couch RB, Englund JA, Whimbey E. Respiratory viral infections in immunocompetent and immunocompromised persons. Am J Med 1997; 102 Suppl: 2-9.

5. Harrington RD, Hooton TM, Hackman RC et al. An outbreak of respiratory syncytial virus in a bone marrow transplant center. J Infect Dis 1992; 165: 987-993.

6. Anderson DJ, Jordan MC. Viral pneumonia in recipients of solid organ transplants. Semin Respir Infect 1990; 5: 38-49.

7. Apalsh AM, Green M, Ledesma-Medina J et al. Parainfluenza and influenza virus infections in pediatric organ transplant recipients. Clin Infect Dis 1995; 20: 394-399.

8. Ljungman $\mathrm{P}$, Andersson $\mathrm{J}$, Aschan $\mathrm{J}$ et al. Influenza $\mathrm{A}$ in immunocompromised patients. Clin Infect Dis 1993; 17: 244-247.

9. Sable CA, Hayden FG. Orthomyxoviral and paramyxoviral infections in transplant patients. Infect Dis Clin North Am 1995; 9: 987-1003.

10. Englund JA, Piedra PA, Jewell A et al. Rapid diagnosis of respiratory syncytial virus infections in immunocompromised adults. J Clin Microbiol 1996; 34: 1649-1653.

11. Lewinsohn DM, Bowden RA, Mattson D, Crawford SW. Phase I study of intravenous ribavirin treatment of respiratory syncytial virus pneumonia after marrow transplantation. Antimicrob Agents Chemother 1996; 40: 2555-2557.

12. Rodriguez WJ, Hall CB, Welliver R et al. Efficacy and safety of aerosolized ribavirin in young children hospitalized with influenza: a double-blind, multicenter placebo-controlled trial. $J$ Pediatr 1994; 125: 129-135.

13. Whimbey E, Champlin RE, Englund JA et al. Combination therapy with aerosolized ribavirin and intravenous immunoglobulin for respiratory syncitial virus disease in adult bone marrow transplant recipients. Bone Marrow Transplant 1995; 16: 393-399.

14. DeVincenzo JP, Leombruno D, Soifer RJ, Siber GR. Immunotherapy of RSV pneumonia following bone marrow transplantation. Bone Marrow Transplant 1996; 17: 1051-1066.

15. Whimbey $\mathrm{E}$, Couch $\mathrm{R}$, Englund $\mathrm{J}$ et al. Respiratory syncytial virus pneumonia among hospitalized adult patients with leukemia. Clin Infect Dis 1995; 21: 376-379.

16. Whimbey $\mathrm{E}$, Champlin RE, Couch RB et al. Community respiratory virus infections among adult bone marrow transplant recipients. Clin Infect Dis 1996; 22: 778-782.

17. Raad I, Abbas A, Whimbey E. Infection control of nosocomial respiratory viral disease in the immunocompromised host. $\mathrm{Am}$ $J$ Med 1997; 102 Suppl: 48-52.

18. Hayden FG. Prevention and treatment of influenza in immunocompromised patients. Am $J$ Med 1997; 102 Suppl: $55-60$. 